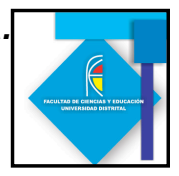

\title{
A PROPÓSITO DE LOS CONCEPTOS DE VELOCIDAD Y RAPIDEZ: UNA PROPUESTA DE FORMALIZACIÓN EN LA ENSEÑANZA DE LA FÍSICA DE ESTOS CONCEPTOS DESDE LA PERSPECTIVA GALILEANA.
}

\author{
CONCEPTS ABOUT SPEED AND SPEED: A PROPOSAL \\ FOR FORMALIZING THE PHYSICS TEACHING \\ THESE CONCEPTS FROM THE GALILEAN PERSPECTIVE.
}

\author{
Rusbley Yadit Osorio Quintero ${ }^{1}$ \\ Yirsen Aguilar Mosquera²
}

\section{Resumen}

En los análisis realizados en investigaciones educativas sobre enseñanza de las ciencias, se hacen evidentes algunas dificultades que los alumnos presentan en los modos de conceptualizar los fenómenos físicos de la velocidad y la rapidez, en las explicaciones que dan los estudiantes sobre el movimiento no se establece diferencia entre estos conceptos. Esto resulta problemático cuando tratan situaciones relacionadas con el Movimiento Circular Uniforme, donde es importante establecer la diferencia entre estos conceptos.

Con la intención de construir rutas conceptuales alternativas para la enseñanza del movimiento, se realiza un análisis histórico epistemológico de la manera como Galileo configura y presenta los conceptos de velocidad y rapidez, expuesto en su obra titulada Consideraciones y Demostraciones Matemáticas (1976) sobre Dos Nuevas Ciencias. En este análisis se muestra cómo Galileo establece una diferencia entre la velocidad y la rapidez, considera a la velocidad como una magnitud intensiva y a la rapidez como magnitud extensiva. Se analiza además cómo el uso de la Geometría en la organización del fenómeno del movimiento que permite a Galileo dar forma a esta clase de fenómenos, aspecto que se constituye en una fructífera manera de significar la relación entre la física y las matemáticas.

Palabras clave: Velocidad, Rapidez, Formalización, Enseñanza, Galileo y Movimiento.

\footnotetext{
${ }^{1}$ Estudios Culturales sobre las Ciencias y su Enseñanza - ECCE-Facultad de Educación, Universidad de Antioquia. Medellín, Colombia.rusandy77@hotmail.com,

${ }^{2}$ Estudios Culturales sobre las Ciencias y su Enseñanza - ECCE-Facultad de Educación, Universidad de Antioquia. Medellín, Colombia.rusandy77@hotmail.com, yirsena@yahoo.es
} 


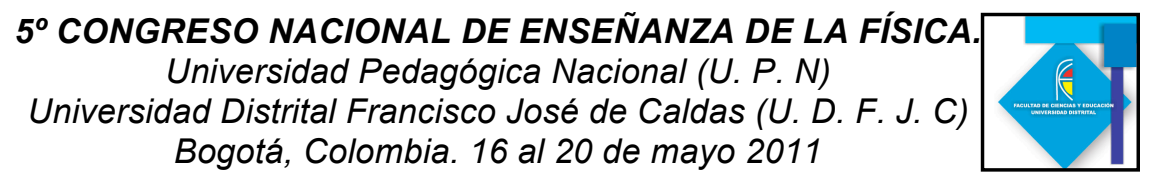

\begin{abstract}
Abstrac
In the analysis made in educational research on science teaching are evident difficulties students have in the ways of conceptualizing the physical phenomena of the speed and quickness, in the explanations given by the students of the movement is not set difference between these concepts. This is problematic when dealing with situations involving uniform circular motion, which is important to distinguish between these concepts.

With the intention to build conceptual alternative routes to teaching movement is a historical analysis of how epistemological Galileo set and introduces the concepts of speed and quickness, as set out in his book Mathematical Considerations and Demonstrations (1976) on Two New Science. This analysis shows how Galileo makes a distinction between the speed and quickness, regarded as a magnitude speed-intensive and extended the speed and magnitude. It also examines how the use of geometry in the organization of the phenomenon of movement that allows Galileo to shape this kind of phenomena, an aspect which constitutes a fruitful way to signify the relationship between physics and mathematics.
\end{abstract}

Keywords: Speed, Speed, Formalization, Education, Galileo and Movement.

\title{
Los conceptos de velocidad y rapidez en el contexto de la enseñanza.
}

Es muy marcado el hecho de que el aprendizaje de las ciencias, en torno a las conceptualizaciones sobre velocidad y la rapidez, encierra la dificultad de que las dos conceptualizaciones aparecen en las explicaciones como dos conceptos sinónimos "para los profesores el concepto de velocidad (o rapidez) parece tan simple y evidente que parece casi imposible que los estudiantes puedan llegar a tener problemas en su comprensión" (Martinez,1999),aun así cabe, dentro del marco epistemológico e histórico, indagar en cómo están construyendo los estudiantes dichos conceptos, pues en muchas ocasiones los estudiantes han recibido las conceptualizaciones adecuadas. No obstante los esfuerzos por diferenciar dichas conceptualizaciones no han logrado menguar las dificultades que persisten en la construcción del conocimiento sobre el saber científico en torno a la velocidad y la rapidez, sin embargo, a pesar de la importancia que tales conceptos tienen, diversas investigaciones realizadas han mostrado que una gran parte de los estudiantes llegan al nivel superior con un buen número de errores conceptuales acerca de la velocidad y la rapidez (Rodríguez, Mena y Rubio, 2008). Además, tienen bastantes dificultades cuando deben definir el significado con precisión (Martínez, 1999).

Abordar estos problemas de la enseñanza, desde una perspectiva histórico epistemológica puede darle otro sentido a estas conceptualizaciones en tanto que está presenta. la necesidad de concebir el aprendizaje como un proceso de construcción activa de significados en el que las concepciones intuitivas o espontaneas de los alumnos desempeñan un papel trascendental en la reconstrucción y recepción de conocimientos elaborados por la comunidad científica. (Driver, 1981). 


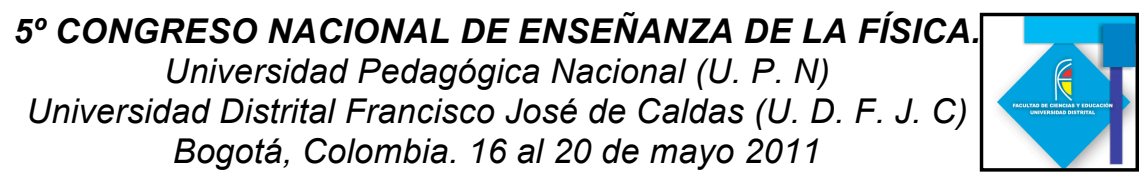

\section{La Historia y la Epistemología en la enseñanza de las ciencias}

La historia y epistemología de las ciencias en la enseñanza de las ciencias permite un acercamiento a las condiciones sociales, políticas, culturales y tecnológicas que han hecho posible el saber sobre las ciencias. Es una nueva tradición filosófica que transforma la mirada del quehacer científico. Esta nueva filosofía de las ciencias permite recrear en un contexto actual la forma en cómo se han logrado los avances en el pensamiento. Va mas allá del simplismo de mostrar qué se ha hecho en la producción del conocimiento científico, para entrar a indagar cómo han sido los debates y, las implicaciones que estos han tenido para determinar la naturaleza de un saber.

Por consiguiente se hace preciso que esta mirada hacia el saber científico traiga con si la intención de que el estudiante sea consciente del saber sobre las ciencias que cree estar adquiriendo en tanto que logre resolver cuestiones científicas y reflexiones frente a estas.

\section{Los conceptos de velocidad y rapidez desde la perspectiva galileana.}

\section{Establecimiento de condiciones iníciales}

Galileo Galilei construye su teoría de la relatividad del movimiento basándose en la formalización de los conceptos de velocidad y rapidez, a través de la perspectiva de la observación y experimentación mental; es por ello que la formalización en la estructura de dichos conceptos juega un papel importante, en tanto que la formalización se caracteriza por ser un proceso cognoscitivo, a través del cual se da forma a los modos de pensar, para así poderlos caracterizar, reconocer, comprender y ser capaz de explicarlos de manera coherente y fundamentar con ello la realidad o los diferentes modos de ver el mundo.

Con lo anterior, Galileo proyecta unas generalidades que caracterizan al movimiento que explica, satisfacen y cumplen con las leyes de la naturaleza, para explicar que el movimiento posee propiedades no medibles y/o cuantificables, y a partir de las cuales se extrae un trabajo que satisface las necesidades de entenderlo en su plenitud. Parte de unas definiciones las cuales examina con detalle y minuciosidad, estableciendo un debate con cada una de las explicaciones que considera claves en la conceptualización; propone axiomas y teoremas a partir de los cuales demuestra las relaciones geométricas con las que explica la validez física de su análisis formal (Wartofsky, M. 1968) ) y establece las condiciones iníciales que determinan el fenómeno; la ocurrencia del movimiento y la organización teórica de su matematización, logrando con ello organizarla bajo una estructura lógica formal que permite examinar sus grados de validez al jerarquizarlas (Aguilar, Y y otros. 2008). 


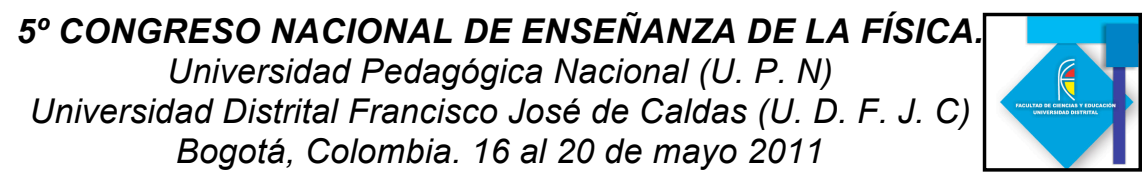

La actividad de axiomatizar, es considera para la postulación de verdades irrefutables, que proporcionan relaciones entre las magnitudes de velocidad, tiempo y espacio,

propiciando a partir de ellos la construcción equivalente de dichos axiomas de modo que se logra estructurar una formulación matemática del movimiento.

El empleo de figuras geométricas como ayuda para razonar constituyó una innovación importante" (Wartofsky, M. 1968) en tanto que se logra a partir de ellas interpretar conceptos cuantificables del movimiento como la velocidad. Las prácticas observacionales llevadas a cabo a través de la idealización le permite la representación geométrica, la cuantificación y transformación de lo abstracto, en tanto que se produce un cambio en la representación y expresión lingüística de la geometría. Con ello Galileo teoriza a la velocidad como la calidad del movimiento susceptible de ser medida como más o menos, en términos de intensidad en tanto que no se le puede atribuir una estructura aditiva y por ello es considerada como una cualidad del movimiento y caracterizándose entonces como una magnitud intensiva del movimiento, lo que permite considerarla como una variable de estado.

De otro lado la rapidez se aborda como la cantidad de movimiento, las cantidades pueden ser aditivas y medibles y por ello la rapidez es una magnitud extensiva y numerable.

\section{El papel de la geometría en la formalización del movimiento}

Una nueva forma de representar el lenguaje geométrico fue el algebraico, en donde las magnitudes del movimiento se comenzaron a significar mediante símbolos. Galileo formulo aportes a la observación desde la geometrización en cuestiones de la calidad de movimiento, pero a través del tiempo estas magnitudes cualitativas se han ido transformando a llegar a ser cantidades de movimiento. Es por ello que se puede deducir que la velocidad representa la cualidad de dicho movimiento y la rapidez la cuantificación de esta cualidad.

Finalmente, es lícito plantear que en la perspectiva galileana se puede interpretar una relación de constitución entre la física y la matemática. En los planteamientos hechos se puede ver como el componente matemático emerge en el proceso de formalización del movimiento, pero a su vez la formalización del movimiento es mediatizada por la construcción de las estructuras matemáticas (geometrización).

\section{Referencias bibliográficas}

Aguilar, M. Ayala, M. Romero, A. Malagón, J. Rodriguez, O. Garzón, M. 2008.Los procesos de formalización y el papel de la experiencia en la construcción del conocimiento sobre los fenómenos físico. Ed Kimpres. Bogotá. 


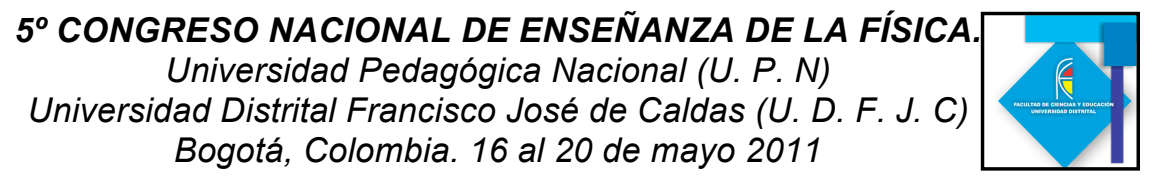

Galilei, G. (1976). Consideraciones y demostraciones matemáticas

sobre dos nuevas ciencias. Editora nacional Madrid España.

Martínez. B. (1999) Los conceptos de velocidad media e instantánea en secundaria y la universidad. Rev Actes III jornades de la curie. Pag 42.

Rodríguez, M., Mena, D., Rubio, C., (2008), Superación de errores conceptuales de ingeniería química industrial cuando estudian la asignatura de física. Tecnología, ciencia y educación, ene-jun. Año 2008/ vol 23 numero 001. Instituto mexicano de ingenieros químicos. Pág.43

Wartofsky, M. (1968). Introducción a la historia de la ciencia 2. Madrid. Ed. Cas: Alianza. 